\title{
Gradhiva
}

\section{Le contexte politique et culturel des droits intellectuels}

\section{Philippe Aigrain}

\section{(2) OpenEdition}

\section{Journals}

\section{Édition électronique}

URL : http://journals.openedition.org/gradhiva/1900

DOI : 10.4000/gradhiva. 1900

ISSN : 1760-849X

\section{Éditeur}

Musée du quai Branly Jacques Chirac

\section{Édition imprimée}

Date de publication : 24 novembre 2010

Pagination : 158-174

ISBN : 978-2-35744-029-6

ISSN : 0764-8928

\section{Référence électronique}

Philippe Aigrain, « Le contexte politique et culturel des droits intellectuels », Gradhiva [En ligne], 12 | 2010, mis en ligne le 24 novembre 2013, consulté le 02 mai 2019. URL : http:// journals.openedition.org/gradhiva/1900 ; DOI : 10.4000/gradhiva.1900 


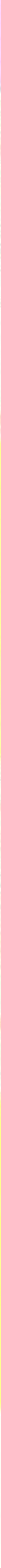




\section{Le contexte politique et culturel des droits intellectuels}

Philippe Aigrain

Fig. 1 Disques publicitaires vinyle en couleur. Collection Daniel Lesueur.

\footnotetext{
-

1. Cause commune : l'information entre bien commun et propriété (Paris, Fayard [《Transversales »], 2005] et Internet et création: comment reconnaître les échanges sur Internet en finançant la création? (Cergy-Pontoise, InLibroVeritas, 2008; http://paigrain.debatpublic. net/?page_id=171).
}

À propos...

Si le copyright des textes écrits jouit d'une longue histoire, celui de la musique ne fait guère l'objet d'une attention spécifique avant les années 1970 (voir l'article de Pierre-André Mangolte dans ce numéro). Depuis 1877, on peut en principe enregistrer celle-ci et la reproduire, mais la reproduction des enregistrements eux-mêmes reste une technique extrêmement coûteuse et délicate. À cet égard, l'apparition des cassettes marque un changement. Doté du cassettophone approprié, n'importe qui peut créer des doubles afin de les vendre ou simplement de partager sa passion avec d'autres. Il reste cependant des limites. D'une part, le procédé s'accompagne d'une perte de qualité, chaque copie ajoutant du "souffle » à l'original. D'autre part, il prend du temps, autant que l'original pour une copie de vinyle à cassette, parfois un peu moins pour une copie d'une cassette à une autre. Enfin, la circulation des cassettes reste largement liée à la rencontre physique de leurs utilisateurs.

On mesure aisément l'écart avec la situation actuelle. Il suffit d'un ordinateur bas de gamme et de quelques minutes pour extraire le contenu d'un disque compact, l'envoyer vers un baladeur numérique, un réseau de partage, ou encore le récupérer pour le graver à nouveau sur un autre disque. La multiplication peut se faire à l'infini, sans perte de qualité (ou avec une perte négligeable pour la plupart des auditeurs). Le disque lui-même devient un support obsolète, à ceci près qu'il reste le moyen privilégié d'offrir de la musique ou de manipuler en direct les enregistrements à des fins créatives (voir l'article de Guillaume Kosmicki dans ce numéro).

Pour Philippe Aigrain, ces développements technologiques sont au cœur d'un débat de société dont l'issue est incertaine et les enjeux importants. Docteur en informatique théorique, habilité à diriger des recherches, il est l'auteur de deux livres $^{1}$ et de nombreux articles. Il est engagé dans une réflexion de fond sur les 
enjeux politiques, sociaux et culturels des techniques informationnelles. Il a contribué à fonder le collectif La Quadrature du net, et participe aux comités directeurs du Software Freedom Law Center et du NEXA Center for Internet and Society. Il dirige par ailleurs la société Sopinspace, qui développe des logiciels libres et fournit des services commerciaux pour l'organisation de débats publics et d'activités collaboratives sur Internet.

Aux approches considérant la propriété intellectuelle comme un point de départ a priori, Philippe Aigrain oppose la nécessité de garantir des «droits intellectuels positifs ». La dimension de ces droits dépasse la musique, tout comme la conception restrictive à laquelle ils s'opposent. Par ailleurs, le copyright musical entretient un lien particulier avec les techniques numériques, et ce pour deux raisons au moins. D'une part, ces dernières sont centrales dans les débats qui défraient régulièrement la chronique. Ce sont elles qui donnent lieu aux accusations de piratage pour des actes qui paraissent tout naturels à leurs auteurs (copier un disque, envoyer une chanson en $\mathrm{mp} 3$, la poster sur un blog, etc.). D'autre part, il a fallu attendre les développements technologiques des quarante dernières années pour que le copyright musical devienne un réel objet de réflexion, tant pour les juristes que pour les anthropologues.

C'est pourquoi il est difficile de penser une ethnographie du copyright sans prendre en considération les enjeux particuliers issus du développement des techniques informationnelles. Le texte de Philippe Aigrain en donne ici un aperçu ${ }^{2}$, et propose un modèle de développement qui témoigne de la richesse de ce débat.

\section{Qu'est-ce que l'information?}

Les marchandises industrielles sont produites sous forme d'unités discrètes, identifiables, échangées et vendues, consommées et utilisées comme un pain ou une automobile. On achète les produits d'un fournisseur et on en prend possession. L'échange est régi par les règles juridiques propres aux contrats. Mais l'information et les connaissances ne sont pas consommées ou «utilisées». Elles sont une production sociale et la question de leur prix ou de leur valeur est très différente de celle des biens industriels.

$[\ldots]$

Comme je l'ai signalé plus haut, l'information est par nature un bien collectif et non un bien privé, une propriétés.

Daniel Bell, préface à la réédition de 1976 de The Coming of Post-Industrial Society («Vers la société post-industrielle»), New York, Basic Books, 1973.

Cette citation vieille de trente-quatre ans nous rappelle cruellement le délai nécessaire à l'assimilation de changements profonds, même lorsque leur nature a été élucidée par des pionniers. L'information au sens moderne résulte de la conjonction de trois éléments :

- la possibilité de représenter des phénomènes et contenus divers «en information » (c'est-à-dire en pratique par des données codées sous forme de nombres) et à l'inverse d'interpréter cette information pour la traduire en textes, sons, images, actions dans le monde physique, etc.;

- la possibilité de programmer et faire exécuter sur des machines " universelles » des traitements de cette information dans le domaine informationnel: ainsi on peut détecter une variation du génome d'un individu ou modifier une image (par exemple la rendre plus contrastée) par un traitement de leur représentation numérique;

2.Ce texte est utilisable selon les termes de la licence Creative Commons By-SA, http:// creativecommons.org/licenses/ by-sa/2.0/fr.

3. Ma traduction. 
- la possibilité d'échanger de l'information sur un réseau universel qui la transmet comme pure information.

On aura reconnu la numérisation, l'informatique et Internet. De ces trois éléments, c'est le deuxième qui fonde les autres.

Qu'est-ce qui change avec les techniques informationnelles?

L'ÉCONOMIE DE LA COPIE : SORTIE DE L'ÈRE DES INDUSTRIES DE LA REPRODUCTION

Les débats actuels se centrent sur l'impact des techniques informationnelles sur la copie, dont nous verrons qu'il n'est qu'un épiphénomène de la révolution informationnelle. Ne nous privons cependant pas d'en explorer la nature. Richard Stallman, qui explicita le projet des logiciels libres, résume l'histoire de la copie en y distinguant trois grandes phases.

Avant l'invention de l'imprimerie (à l'ère des manuscrits et des codex), la seule façon de produire une copie d'un texte était... de le recopier. Produire cent copies prenait plus ou moins cent fois plus de temps que d'en produire une. Il n'existait à l'époque aucune réglementation du droit de copie (sauf pour les textes qui avaient le malheur de déplaire aux pouvoirs en place). Avec l'invention de l'imprimerie, produire en masse des exemplaires coûte nettement moins cher

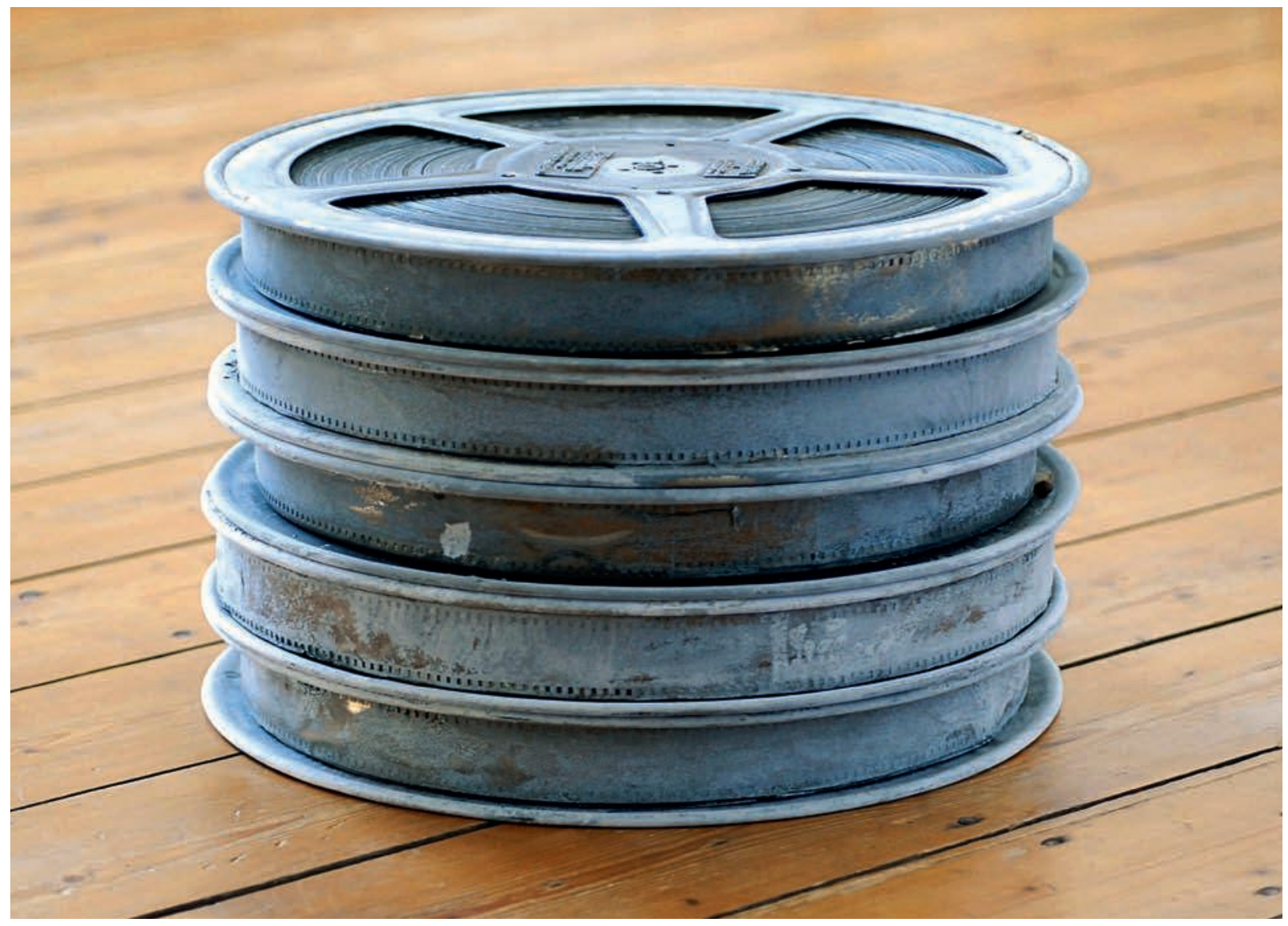

Fig. 3 Joseph Beuys, Das Schweigen, 1973 [Le silence], éd. R. Block, 5 bobines de pellicule zinguées du film @ Édition Block, Berlin/ADAGP, Paris 2010. 
à l'unité que d'en copier un exemplaire. Mais si l'on doit produire simplement une copie, il est moins coûteux de la produire «à la main » que de l'imprimer. Les réglementations qui se mettent progressivement en place dans les deux siècles qui suivent réglementent donc l'activité d'impression et d'édition commerciale sans restreindre d'aucune façon le droit de chacun de copier ce qu'il entend. Avec l'informatique et le numérique, 1,6 milliard de personnes sont dotées de machines qui permettent de produire une copie d'un fichier numérique représentant une œuvre à un coût qui est à peine supérieur à celui de la reproduction en masse. La situation s'est rééquilibrée entre copie à l'unité et reproduction de masse : un million d'internautes produisant chacun une copie concurrencent efficacement un grand éditeur qui en produit un million. On retrouve l'équilibre qui existait à l'époque de l'artisanat manuel de la copie, mais avec un coût unitaire de celle-ci devenu presque nul alors qu'il était très élevé avant l'invention de l'imprimerie.

Dans ces conditions, il n'est possible de maintenir des monopoles de reproduction et de distribution qu'en contraignant les activités de tout un chacun et en privant les citoyens de la planète d'une capacité dont la portée est considérable. Pour beaucoup, cette entreprise est impossible. Les plus précis ajoutent «sauf à attenter de façon insupportable aux libertés et aux droits ». Je soutiendrai pour ma part qu'un tel projet serait obscurantiste et inutile, voire nuisible aux droits fondamentaux des auteurs et des producteurs.

LE CONTINUUM DE POSITIONS ENTRE RÉCEPTION ET PRODUCTION PROFESSIONNELLE

réception

consommation

réception passive recommandation comparaison comment. expression pro-am production création

production

«industrialisation»

Fig. 4 Du face-à-face au continuum de positions.

L'effet le plus remarquable de la diffusion de plus en plus générale des techniques informationnelles est la substitution de continuums de positions aux face-à-face qui caractérisaient l'ère industrielle. La séparation nette entre production industrielle et consommation est remplacée (dans le champ des biens informationnels et d'autres artéfacts où l'information joue un rôle clé) par une situation beaucoup plus complexe, où il existe des positions intermédiaires de contribution ou de véritables modes de production distribuée parmi les pairs. Dans le champ culturel, le fossé entre la création professionnalisée et la réception passive de produits ou services est, de façon encore plus spectaculaire, comblée par des positions intermédiaires.

Cette situation renoue avec l'ère artisanale, mais dans un contexte profondément renouvelé par les technologies. Contrairement aux caricatures promues par les nostalgiques de la culture comme activité séparée d'une élite, les positions extrêmes ne disparaissent en rien : il y a par exemple aujourd'hui plus de créateurs professionnels, plus de musiciens d'une très grande qualité qu'auparavant. La réception "passive» des œuvres ne disparaît pas plus. Elle devient une 


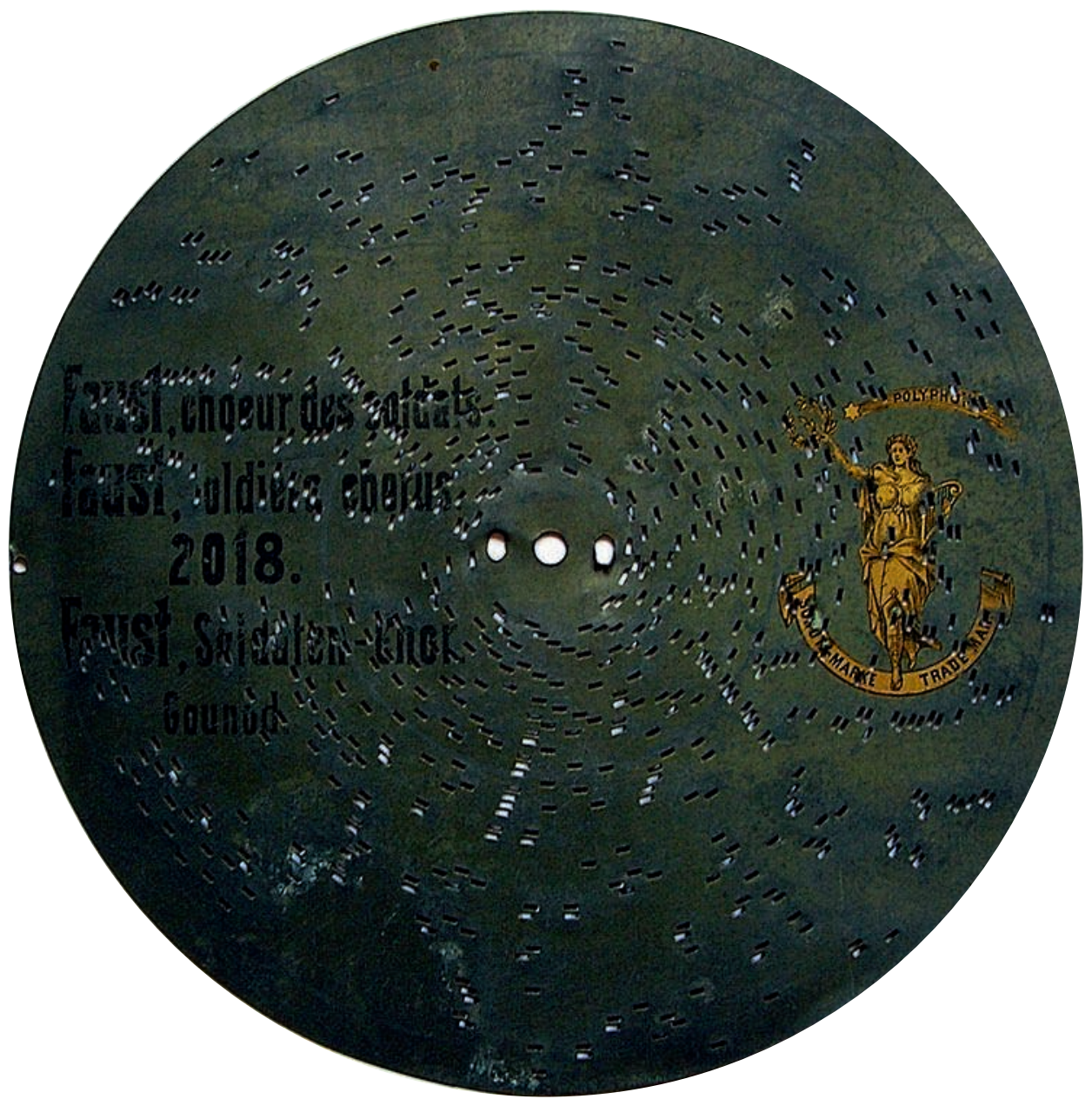

Fig. 5 Disque perforé comportant un mécanisme semblable à celui des boîtes à musique. Collection Daniel Lesueur.

pratique parmi d'autres, alternant avec d'autres formes d'écoute ou de visionnement plus actives, visant un but particulier, de production, de comparaison ou d'analyse4. Mais les positions intermédiaires et la facilité (relativement) accrue à passer d'une position à une autre dans le continuum ci-dessus permet un accès accru à certaines pratiques culturelles : critique, remix, parodies. En outre, les statistiques sur les pratiques culturelles font apparaître un renouveau des pratiques artistiques (y compris traditionnelles) depuis 1995 environ, alors que pendant l'ère des industries de la reproduction leur érosion avait été constante.

-

4. Sur ce point, voir Philippe Aigrain et Philippe Lepain, rapport du groupe Écoute interactive de la musique de la Bibliothèque nationale de France, 1995 et Peter Szendy [éd.], L'Écoute (Paris, L'Harmattan, « Cahiers de l'Ircam », 2000).

5. Voir ici même l'article de Guillaume Kosmicki, ndlr.

6. Voir plus bas $\mathrm{p} 171$

\section{PRATIQUES COLLECTIVES}

La conjonction de l'informatique et des réseaux est constitutive de nouvelles pratiques collectives, comme celles de la musique techno par exemples. La production par les pairs sur la base des biens communs ${ }^{6}$ existant par exemple pour les logiciels libres en est une forme aboutie. Ce n'est pas forcément dans la production d'une œuvre ou d'un objet unique que se développent des pratiques collectives : $80 \%$ des logiciels libres n'ont qu'un auteur. De même, la plupart des articles de Wikipedia n'ont que quelques contributeurs significatifs, d'autres intervenants apportant de petites touches ou corrections à leurs travaux. Comme nous le verrons, la vraie coopération se fait au niveau de l'écosystème d'ensemble des productions. 


\section{SYMÉTRISATION DES MÉDIAS}

Un des aspects particulièrement importants de la fin du face-à-face des producteurs et des consommateurs est la symétrisation des médias. Celle-ci est une réalité technologique qui donne à chacun la possibilité d'être producteur et distributeur de contenus. La tendance peut même s'observer dans des médias comme l'image animée, où ces activités étaient jusqu'ici réservées à un cercle restreint. La symétrisation des médias est encore plus évidente dans le champ des médias « natifs » de l'ère informationnelle comme les blogs.

Se pose donc ici la question : si tout le monde écrit, restera-t-il des lecteurs? Les études actuelles ${ }^{7}$ apportent une réponse positive, et montrent également que subsisteront des auteurs dont les productions attireront une audience importante. Mais l'attention portée aux œuvres sera répartie de façon bien plus diverse que celle qui existe pour les produits des industries culturelles. Les intérêts de plus en plus diversifiés du public nous imposeront de réviser nos attentes : au rêve rarement réalisé d'émarger au succès planétaire se substituera pour beaucoup d'auteurs, artistes et intellectuels la réalité d'un public étendu mais restant de taille modérée...

\section{ÉCOSYSTÈMES CULTURELS RICHES ET INTERDÉPENDANTS}

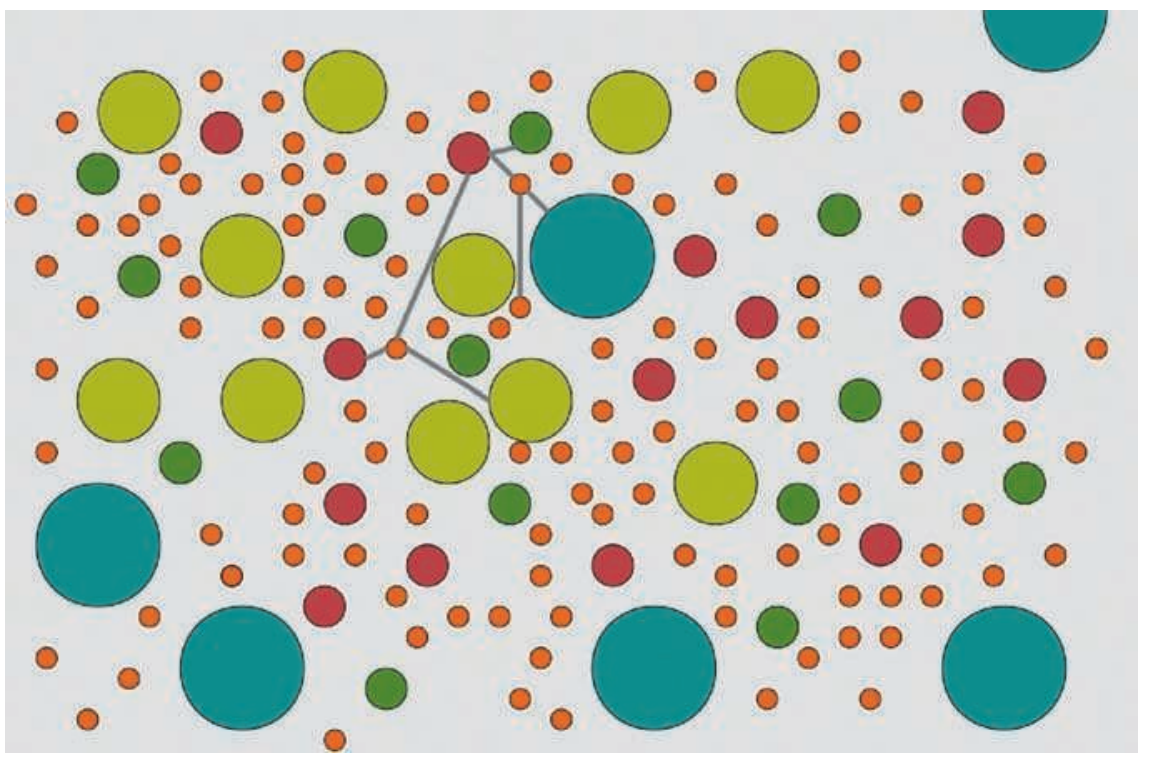

Fig. 6 Des écosystèmes riches et interdépendants.

Comme esquissé précédemment, la réalité de la coopération à grande échelle à l'ère de l'information apparaît surtout lorsqu'on considère non pas des productions individuelles mais des ensembles de productions interdépendantes. C'est le cas du logiciel, dont l'exécution fait appel à des dizaines d'autres logiciels. Ce type d'interdépendance existe également pour la culture, de façon évidente dans le cas du remix, mais aussi par des mécanismes moins immédiats : citation, réutilisation de formes ou de références. On comprend ici pourquoi des limitations (croissantes) mises à l'accès et à la réutilisation conduiraient à un appauvrissement de la création elle-même.
- -

7. Cf. Philippe Aigrain, « Diversity, Attention and Symmetry in a Many-to-Many Information Society $\gg$, First Monday 11(6), juin 2006 (http://firstmonday. org/htbin/cgiwrap/bin/ojs/index. $\mathrm{php} / \mathrm{fm} / \mathrm{article} / \mathrm{view} / 133 \mathrm{P} / 125$ ? et les travaux décrits dans Sharing and Creative Economy: Culture in the Internet Age, édition partielle, 2010 (http://paigrain.debatpublic. net/docs/Internet_creation_1-3.pdf). 


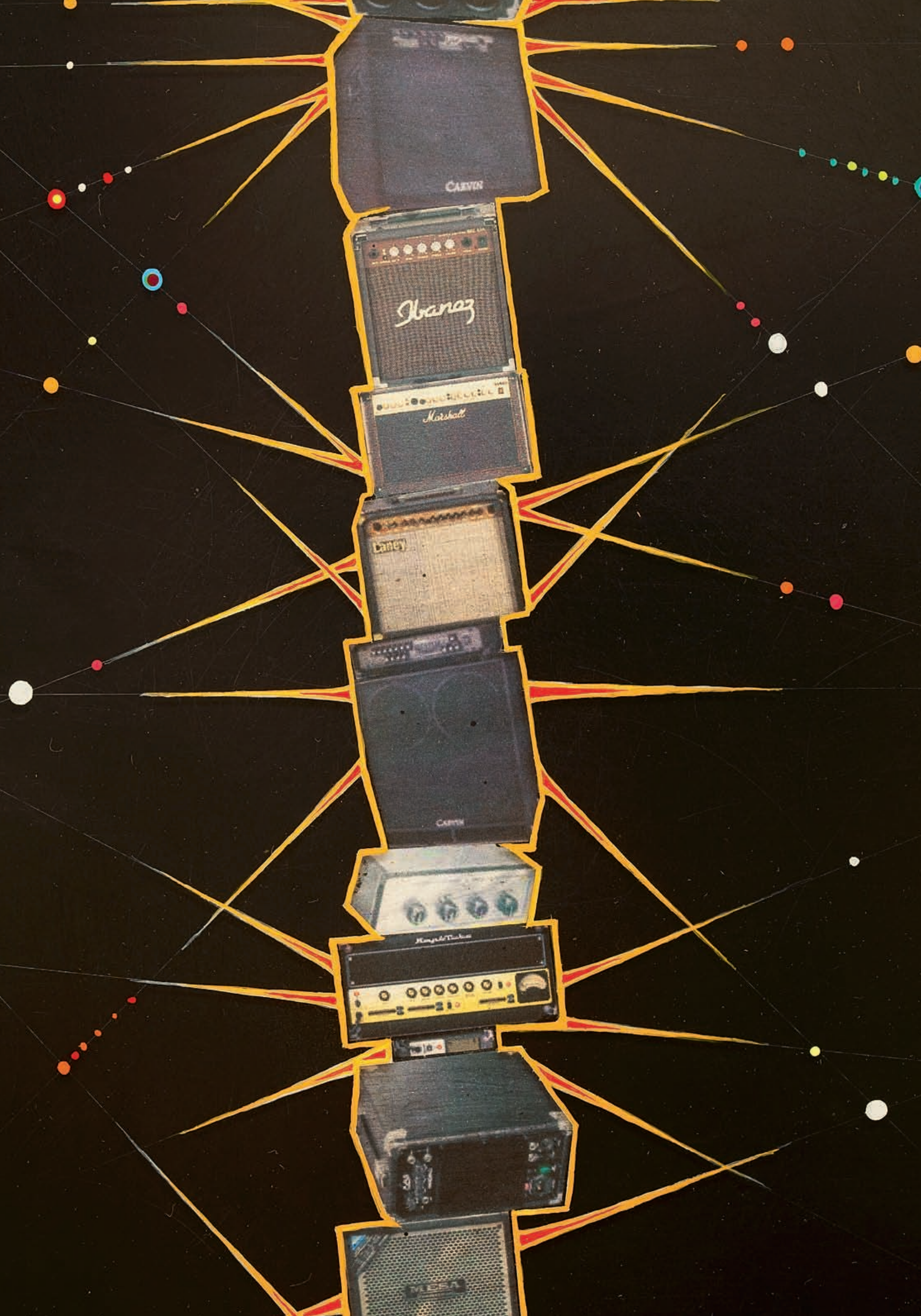




\section{Concentration des médias, marchandisation du temps et dépossession technologique}

Comment en est-on arrivé à ce que l'ère de l'information se traduise, au moins en apparence, par tout l'inverse de ce que je viens de décrire : des restrictions sans précédent des droits d'usage, une extension permanente des mécanismes propriétaires, et ce qui va avec, des monopoles renforcés dans certains secteurs et un pouvoir accru de ceux-ci sur le temps et l'attention des êtres humains? Il y a à cela deux raisons. La première tient au fait que les techniques informationnelles sont universelles et agnostiques : elles servent ceux qui se servent d'elles et, en la matière, les grandes entreprises, la finance et certaines activités des États ont pris de l'avance sur les individus et les groupes sociétaux qui n'y ont eu accès que progressivement à partir des années 1980. De ce fait, les industries informationnelles (médias, édition, certaines biotechnologies, logiciels) se sont emparées des technologies pour renforcer leur contrôle sur les marchés et les usages. Depuis ces dernières années, elles sont cependant sérieusement fragilisées par la concurrence des approches ouvertes à un partage accru des connaissances et de l'innovation, et se sont, de ce fait, agrippées à un cadre juridique restrictif.

La seconde raison réside dans la désastreuse incapacité des décideurs à prendre la mesure des changements en cours, qui s'explique par l'influence des groupes d'intérêt, mais aussi par un véritable fondamentalisme de la propriété. En près de trente ans, on a oublié que les brevets, le copyright et les droits exclusifs liés au droit d'auteur ne sont que des incitations parmi d'autres à créer ou à innover, les subventions, les crédits d'impôt, le statut des enseignants et des chercheurs ou le mécénat ayant toujours joué un rôle au moins aussi important. Leur contribution aux revenus et au financement de la création doit toujours être comparée à leurs effets sociaux corrélatifs : obstacles à l'accès à la culture et à l'éducation, mais aussi difficultés pour les créateurs eux-mêmes à réutiliser les œuvres existantes. Enfin, on a oublié que ces dispositifs propriétaires constituent une exception à la règle des biens communs et du domaine public8.

Par aveuglement ou pour servir des intérêts établis, une course folle a été lancée sur trois fronts : l'extension des mécanismes propriétaires, le durcissement de leur mise en œuvre et la réécriture de l'histoire des droits.

TRENTE-CINQ ANS D'EXTENSION DU PÉRIMÈTRE DES DROITS RESTRICTIFS...

À partir des années 1970, l'application de brevets à de nouveaux domaines comme les molécules, l'information génétique (avec un certain succès) ou encore les logiciels (avec plus de résistance, notamment en Europe et en Inde) s'est mondialisée dans le cadre d'accords commerciaux ou de traités. Cette extension des brevets à l'information y produit des effets beaucoup plus négatifs que dans le domaine des processus ou artéfacts matériels. Elle autorise des prix de monopole découplés du coût de production, ce qui en fait des cibles si tentantes pour les investisseurs qu'une grande part de l'innovation pharmaceutique et agroalimentaire en est biaisée.

L'extension de la logique des brevets n'a pas épargné le copyright et le droit d'auteur puisque, de facto, des sujets qui étaient complètement hors de leur champ d'application s'y sont trouvés inclus. Ce fut notamment le cas du droit de mise à disposition non commerciale (le droit de partager sans but lucratif une
PAGE CI-CONTRE

Fig. 7 Fred Tomaselli, Stack, 2008, acrylique, photocollage, résine sur panneau de bois, (détail) (c) Fred Tomaselli. Avec l'aimable autorisation de la James Cohan Gallery, New York/Shanghai.

DOUBLE PAGE SUIVANTE

Fig. 8 André Avelãs, Earphones, installation dans les sous-sols du Smart Project Space Building, DNK's Post Digital Festival, Amsterdam, 2008 @ André Avelãs/photo Paul Glazier.
8. Voir le site http://www.
publicdomainmanifesto.org 


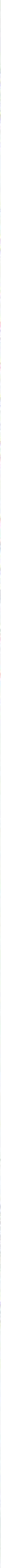


$\because \cdots \div$

$7 \frac{2}{\cdots} \div$
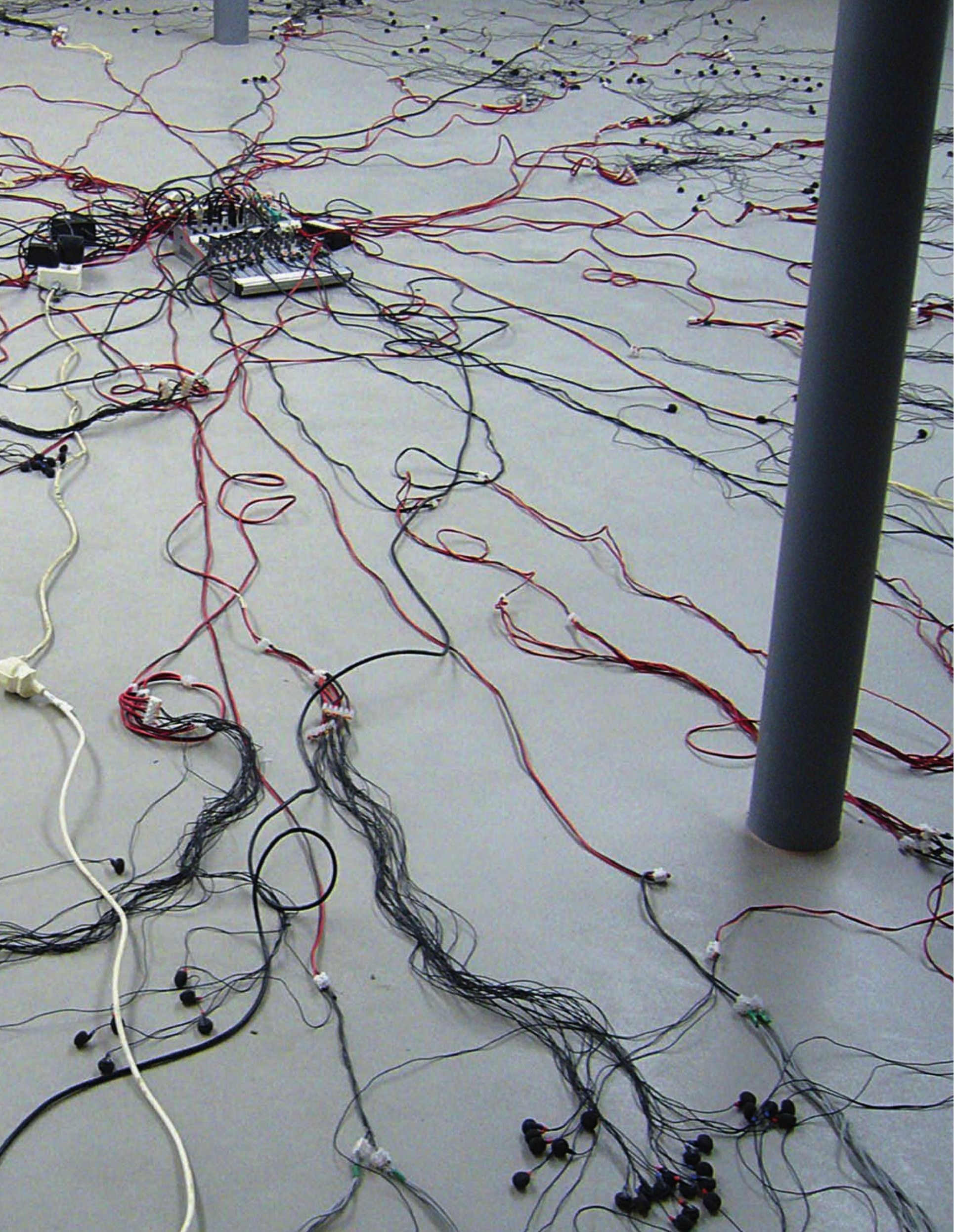
- -

9. Ce sont notamment la présomption d'innocence et le droit à un procès équitable qui sont aujourd'hui menacés. En effet, on est passé d'une constatation judiciaire a posteriori d'une éventuelle violation du droit d'auteur, effectuée par un tribunal après un procès contradictoire, à la multiplication de mécanismes de contrôle ou de répression automatique : mesures techniques empêchant a priori de commettre certains actes, polices privées des ayants droit surveillant les communications personnelles pour y détecter ce qu'elles considèrent comme des infractions, autorités administratives jouant le rôle de boîtes aux lettres entre ces polices privées et le parquet, ordonnances pénales et juge unique privant le processus judiciaire de son caractère équitable.

10. James Boyle, «A politics of intellectual property: Environmentalism for the Net? », Duke Law Journal 87, 1997 ; http:// www.law.duke.edu/shell/cite. pl?47+Duke+L.+J.+87+pdf.

11. «Droits intellectuels positifs et échanges d'information », http ://www.freescape.eu.org/ biblio/article.php3? id_article $=133$

12. http://fr.wikipedia.org/wiki/ Res_communis. information ou une œuvre protégée). En effet, à l'époque de la naissance du droit d'auteur, ce que le détenteur individuel de la copie d'une œuvre en faisait, hors de toute activité commerciale, était hors du champ du droit d'auteur. On est revenu depuis sur cette liberté lorsqu'il est apparu qu'à l'ère du numérique elle aurait des conséquences beaucoup plus importantes. En prime, de nombreux dispositifs propriétaires ad hoc ont été créés, comme celui qui institue des monopoles propriétaires en Europe pour les bases de données, auparavant considérées comme de simples collections de données uniquement soumises au droit d'auteur ou au copyright dans le cas où la nature de la collection constituait une création suffisamment originale.

\section{... DE DURCISSEMENT DE LA MISE EN EUURE DES DROITS}

Les techniques informationnelles fragilisant toujours plus l'exécution pratique des droits restrictifs, on a refusé de questionner la pertinence de ce projet et l'on a sans cesse durci la surveillance, le contrôle technologique et les sanctions pour tenter de transformer en réalité un rêve de dictateur planétaire : conserver comme monopole pour quelques-uns une capacité qui est désormais à la portée d'un quart de la population mondiale. Rien ne semble pouvoir arrêter cette tendance : ni le ridicule de son inefficacité, ni les incroyables atteintes aux droits dont elle est porteuse ${ }^{9}$, ni le fait qu'elle conduise nos démocraties à mettre en place des dispositifs technologiques et administratifs qui semblaient réservés aux États autoritaires.

\section{... ET DE RÉÉCRITURE DE L'HISTOIRE DES DROITS}

Comme tout fondamentalisme, celui des droits intellectuels restrictifs inclut une réécriture du passé. Ainsi le droit reconnu de partager les œuvres sur support (qui permet de donner, prêter et même louer ou vendre un livre, un disque ou un DVD) est-il devenu un précédent à éradiquer. Celui-ci est redéfini comme tolérance due à l'impossibilité passée d'empêcher ces actes, ou supprimé par des attaques contre les bibliothèques et les médiathèques de prêt. Tout cela pour éviter de se demander si, plutôt que d'empêcher l'extension du droit au partage aux œuvres informationnelles, il ne vaudrait pas mieux l'organiser.

\section{Comment s'outiller?}

Dans cette situation, un appareillage conceptuel adéquat est essentiel. Je propose trois pistes à cet effet.

\section{DROITS INTELLECTUELS POSITIFS (DROITS À OBTENIR DES RÉSULTATS, CAPACITÉS)}

La première étape relève de l'hygiène mentale. Elle consiste à penser à ce que l'on essaie d'obtenir et à définir donc des droits intellectuels positifs, des droits à obtenir des résultats ou donnant à leurs détenteurs de plus grandes capacités à agir. Sur les traces de James Boyle $e^{10}$, j'ai proposé cette approche dans un article publié en $2000^{11}$. Il s'agit de renverser la perspective afin de retrouver la primauté de la res communis ${ }^{12}$ pour l'information et les connaissances. 
Le rôle central et premier des biens communs, ce qui appartient à tous et ne peut donc devenir la propriété de personne (pas même de l'État qui ne peut s'en instituer que le garant), est à la base de la refondation des droits intellectuels. Il est aussi la source de la réinvention du politique à notre époque. Les droits de chacun quant aux biens communs doivent être effectifs. Il faut pour cela protéger ces derniers contre la réappropriation, qu'elle résulte de l'extension du périmètre de la propriété ou d'acteurs qui se les approprient de fait. Ce dernier cas se présente par exemple lorsque des œuvres du domaine public sont en pratique inaccessibles ou ne sont pas librement utilisables parce que les seules éditions sont propriétaires du fait d'ajouts marginaux, tels les appareils critiques, ou que les bibliothèques et archives publiques ne les rendent pas accessibles ou le font sous des termes d'usage restrictifs. Ainsi, les grandes bibliothèques patrimoniales soumettent-elles dans la plupart des cas les versions numérisées de documents du domaine public à des termes d'usage soumettant l'usage commercial ou la reproduction publique à des autorisations et royalties.

Mais l'importance de la reconnaissance des biens communs ne s'arrête pas là. Ils instituent des relations entre producteurs, usagers et œuvres qui diffèrent de celles qui sont supposées exister lorsqu'on se situe dans un modèle d'autorisation propriétaire.

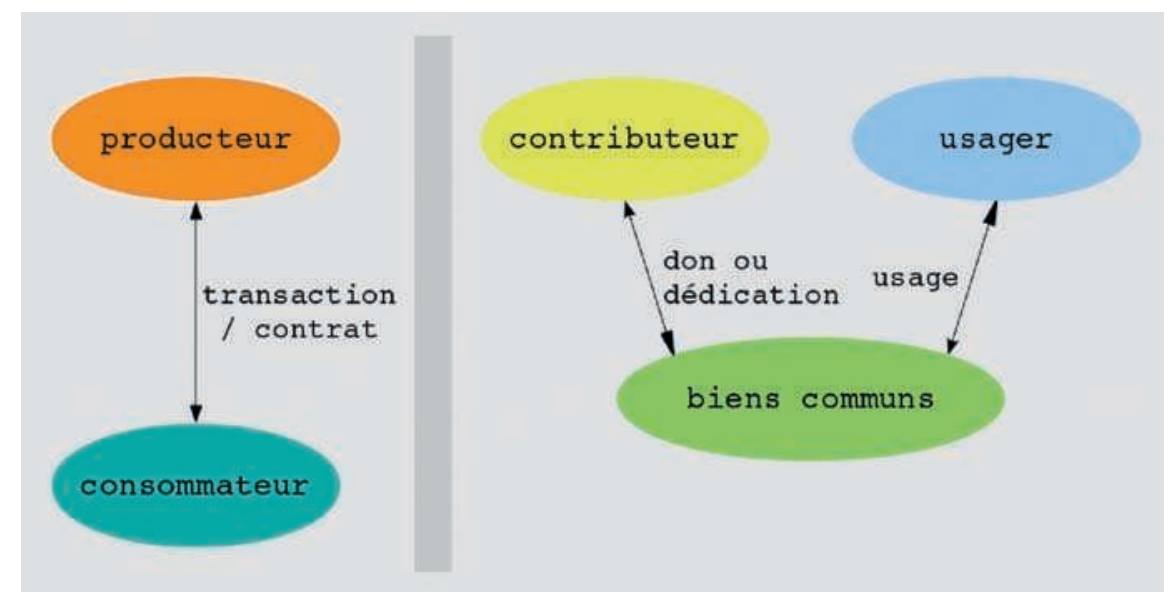

Fig. 9 Le caractère ternaire des communs.

Le modèle des contrats et des transactions monétaires éventuellement associées est binaire : un producteur ou un ayant droit y passe un contrat avec un usager, contrat qui délimite une île de permission dans un océan d'interdictions. Ce modèle est une grossière simplification de la réalité de la loi. Il existe de nombreuses activités qui sont permises sans l'autorisation de l'ayant droit, par exemple la citation, la parodie ou le fait de jouer un disque à la radio (avec une rémunération dans ce dernier cas). Le problème est toutefois que ces activités sont décrites (en Europe continentale) comme des exceptions ou limitations par rapport à la situation de référence. 
Lorsqu'un statut de biens communs est attribué à des productions, la situation est bien différente : le contributeur fait don ou dédie ce qu'il a produit aux biens communs; l'usager prend des biens communs. Le contributeur peut avoir certains droits sur ces biens (par exemple la reconnaissance du fait qu'il y a contribué) et l'usager certains devoirs (notamment celui de conserver le même statut aux biens que lui-même crée par copie, adaptation, etc.). Ces droits et devoirs ne sont pas l'objet d'une transaction directe entre le contributeur et l'usager ${ }^{13}$. Absence de transaction ne signifie pas absence de relation, bien au contraire. L'étude empirique des communs a montré que la reconnaissance, l'attribution et les liens entre contributeurs et usagers y sont plus forts que dans la situation transactionnelle ${ }^{14}$. Les biens communs informationnels sont le lieu d'une réciprocité collective (non contraignante) sur la base de laquelle des reconnaissances entre personnes se développent facultativement. Ils sont porteurs de nouvelles figures du collectif ne reposant pas sur l'appartenance immuable mais sur la participation. Comme l'a écrit Yochai Benkler, «les communs sont un espace institutionnel où nous sommes libres ", mais cette liberté n'exclut pas la convention, l'étiquette, les bons usages, la civilité15.

\section{REPENSER LES DROITS ET LIBERTÉS}

Libérés d'un corset idéologique, ayant reconnu la valeur et le potentiel des communs, tout reste à faire, notamment en ce qui concerne la création de leurs conditions d'existence. C'est une chose de savoir qu'il n'y a pas de fatalité de la tragédie des communs et bien au contraire un grand risque de tragédie des enclosures, c'en est une autre de savoir comment créer l'environnement économique et social des communs créatifs et d'innovation; comment y assurer une justice sociale, dans un pays ou dans le monde. Dans ce qui suit, j'esquisse des pistes de travail dans trois cas qui me paraissent particulièrement importants et proches des préoccupations des anthropologues de la musique.

\section{Libertés d'usage hors marché et financement mutualisé de la création numérique}

recourir à ce modèle en l'absence de définition juridiquement effective de ce qu'est un bien commun institué volontairement.

14. Voir Milad Doueihi, La Grande Conversion numérique. Paris, Seuil, 2008.

15. Voir Milad Doueihi, Ibid.

16. Richard Stallman, « The right way to tax DAT », Wired Magazine, mai 1992 ; http://www.gnu.org/ philosophy/dat.html.

17. Voir Philippe Aigrain, Internet et création..., op. cit., et un développement plus approfondi en cours in Philippe Aigrain (avec l'aide de Suzanne Aigrain], Sharing and the Creative Economy: Culture in the Internet Age, publication partielle : http://paigrain.debatpublic.net/ docs/Internet_creation_1-3.pdf.
Nul n'a pu échapper au débat qui oppose les éradicateurs du "piratage» aux défenseurs d'un droit au partage hors marché des œuvres numériques. Depuis 2003 (1992 si l'on revient aux propositions de Richard Stallman dans un contexte un peu différent ${ }^{16}$ ), des propositions ont vu le jour pour servir à la fois les libertés d'usage et les conditions d'existence de la création. L'obstination des gouvernements et des groupes d'intérêt à refuser d'en débattre (ou à les caricaturer) a servi d'aiguillon pour étayer et mûrir ces propositions ${ }^{17}$. Leur principe consiste à mener de front la reconnaissance du droit au partage hors marché entre individus et la mise en place de nouveaux financements mutualisés pour les activités créatives, reposant sur une contribution créative forfaitaire des abonnés à Internet à haut débit. Les sommes collectées serviraient à la fois à rémunérer les auteurs et contributeurs à la création des œuvres, en lien avec leurs usages sur Internet, et à financer de nouvelles activités créatives ou leur environnement. Dans les deux cas, les internautes seraient mobilisés pour fournir, sans intrusion dans leur vie privée, les données servant à la répartition des sommes collectées. Nul ne peut prédire le sort exact que connaîtront ces propositions 
lorsque les pouvoirs politiques et institutionnels en place ${ }^{18}$ accepteront enfin de les étudier et d'en débattre. Mais le fait même qu'elles surnagent avec obstination malgré le refus répété de les explorer est un signe positif.

Le statut des biens communs issus de communautés non universelles

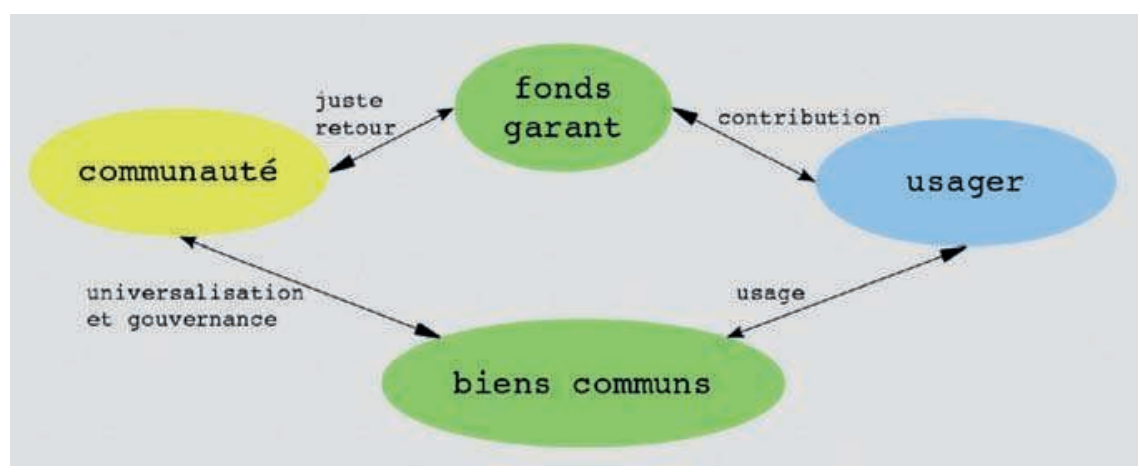

Fig. 10 Des circuits distincts pour le juste retour et les droits d'usage?

C'est avec prudence que j'aborde maintenant un sujet que les anthropologues ont rencontré dans leur pratique, bien avant qu'il ne fasse l'objet de débats dans les enceintes internationales : celui des droits des communautés sur leurs productions culturelles. À l'Organisation mondiale de la propriété intellectuelle (OMPI) et à l'Unesco, cette question est débattue sous le nom de "savoir-faire traditionnel et folklore» dans un panier où l'on trouve également les ressources génétiques. Du fait de son cadre de pensée, l'OMPI a proposé de traiter cette question des droits communautaires par l'attribution de titres de propriété similaires aux brevets ou aux droits exclusifs d'auteur. Il s'est ensuivi de nombreuses critiques de la part des ONG de développement et de pays du Sud. Ces critiques soulignaient que les approches propriétaires ne bénéficient qu'aux puissants qui ont les moyens de faire respecter les restrictions, et que leur usage risquait de donner lieu à des détournements lorsque des acteurs administratifs ou privés s'approprieraient les bénéfices d'accords d'exploitation qu'ils passeraient au nom des communautés.

Peut-on éviter les approches propriétaires en maintenant l'exigence du juste retour pour les communautés lorsque ce qu'elles ont produit (ou ce qu'on en a conservé) est exploité? Le problème est complexe, mais il n'est pas inconcevable de placer les productions ou ressources correspondantes sous un statut de bien commun. Dans bien des cas, cela revient simplement à reconnaître qu'elles y sont déjà. En parallèle, il serait reconnu un droit des communautés à obtenir des revenus ou d'autres formes de bénéfice lorsque les usagers de ces biens communs les utilisent dans des activités économiques ou comme points de départ pour d'autres innovations. Il n'est certes pas aisé de garantir un tel droit dans les rapports internationaux, souvent asymétriques qui plus est. Une solution serait de recourir à un fonds garant, initialement alimenté par les États, puis constitué par des prélèvements sur les bénéfices d'usage (comme illustré dans le diagramme de la figure 10).

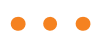

18. Dans divers pays (France, Allemagne, Suède, par exemple), différents partis ou même des sociétés de gestion ont mis des propositions de ce type à leur programme. 
PAGE CI-CONTRE

Fig. 11 Enregistrement d'une séquence musicale de Fantasia de Walt Disney, dirigée par Leopold Stokowski, 1940

(c) Bettmann/Corbis.
Il faut aussi prendre en compte le fait que les retours financiers ou les transferts de technologie ne répondent pas toujours aux préoccupations des sociétés concernées. Certaines estiment que des lieux, des créations ou des activités particulières ne sont, tout simplement, pas utilisables en dehors d'un cadre symbolique qui leur est propre. Il s'agit ici de droits «moraux", ce qui m’amène à un dernier point.

\section{Le devenir des droits moraux}

Les droits moraux sont une noble idée qui sert aujourd'hui principalement à de sordides marchandages, notamment lorsque des héritiers de droits s'en servent pour faire monter la facture des droits patrimoniaux. Ce panier de droits hétérogènes regroupe paternité (attribution), intégrité, repentir, retrait, etc. Lorsqu'il s'agit de les opposer aux intérêts des producteurs et des distributeurs, les droits moraux ont pour l'essentiel perdu toute effectivité : les directives européennes sur la télédiffusion et la transmission par satellite, par exemple, les ont vidés de tout sens en transférant l'exercice pratique de ces droits, soi-disant inaliénables, des auteurs aux distributeurs. Ainsi les conditions de coupures publicitaires dans un téléfilm échappent-elles totalement dans les faits au contrôle de l'auteur. Le droit moral à l'intégrité est aussi questionné pour des raisons plus profondes : dans le monde numérique, la présentation d'une œuvre est de plus en plus sous le contrôle de l'usager final. Je propose de reconsidérer les droits moraux en trois catégories :

- Le droit à l'attribution, reconnu quasi universellement par son inclusion dans la convention de Berne, et qui reste de l'ordre de la loi, sa mise en œuvre s'adaptant aux conditions concrètes des médias et des technologies.

- Les droits comme le respect de l'intégrité et plus généralement de l'intention de l'auteur par rapport à l'usage de l'œuvre, dont la valeur serait reconnue, mais dont le respect ne serait pas confié à la loi mais au registre des normes sociales, des conventions et des bonnes pratiques. Des situations de ce type existent déjà. Ainsi, tout intellectuel qui se respecte honnit la citation hors contexte, mais elle n'est pas prohibée par la loi. Le respect de la prescription, consistant précisément à respecter le sens de ce que l'on cite, est confié à la critique, au jugement des pairs, aux réputations.

- Enfin, le droit au repentir et au retrait a perdu tout sens (s'il en a jamais eu un) dans l'ère de l'information. Il est nécessaire de le remplacer par la capacité concrète de chacun à faire connaître ses opinions présentes et leurs différences par rapport à celles émises dans le passé.

La Quadrature du Net pa@laquadrature.net 
